\title{
A New Cavity Ring-Down Topology for Remote Sensing
}

\author{
D. J. Passos ${ }^{1,2}$, S. O. Silva ${ }^{1}$, M. B. Marques ${ }^{1,2}$, O. Frazão ${ }^{1,2}$ \\ ${ }^{1}$ INESC Porto, Porto \\ ${ }^{2}$ Department of Physics and Astronomy, University of Porto
}

\begin{abstract}
A new, fiber-based, cavity ring-down topology is presented which enables the application of the cavity ring-down technique to remote sensing, by the use of a large cavity ring and an optical circulator. For a proof of concept a $1.5 \mathrm{~km}$ ring is assembled and a taper is used as a sensing head for measuring displacement. The cavity ring-down technique is seen to hold some potential for remote sensing through its implementation on optical fibers.
\end{abstract}

Keywords: Optical Fiber Sensors, Remote sensing, Cavity Ring-Down

\section{Introduction}

The cavity ring down (CRD) spectroscopy is a widely used technology for chemical and molecular analysis in real time [1]. The evolution of this technique led to the development of diverse configurations based on the same underlying principle, i.e., a resonant optical cavity with high reflectivity mirrors.

The recent development of a fiber optic based CRD, which uses a fiber loop as a resonant cavity, has been gaining popularity in the community, for presenting a potential alternative to the usual CRD [2]. In this manner, the conceptual study of the CRD technique became a focus of attention in the area of optical spectroscopy, for the last decade. Only recently this technique has been applied to the measurement of physical parameters, such as: deformation [3-4], pressure [5] and temperature [6].

In this work, the potential of a fiber-based CRD configuration for remote sensing is explored. Previously a configuration with an OTDR as a source of optical pulses and a fiber loop placed after several kilometers of fiber was proposed [7]. Here the authors suggest a different topology in which the ring cavity and all the elements are near the source and remote sensing is made possible by using a large ring cavity, with a sensing head between an optical circulator and a thin film based mirror.

\section{Methodology}

A commercial Optical Time Domain Reflectometer (OTDR), Yokogawa AQ7270, was used to send one microsecond pulses down an optical fiber (SMF 28) which couples to a fiber loop through a 99:1 directional coupler. The fiber loop forms a resonant cavity. Once in the cavity, the pulse will be directed by a circulator through over a kilometer of optical fiber whose end is coated with a gold thin film to form a high reflectivity mirror (M). The pulse reflects at the mirror and returns to the loop. A second 99:1 directional coupler is inserted after (port 3 of) the circulator, connected to a photodetector and an oscilloscope, for monitoring the decay of the pulse. From here is standard cavity ring-down. The pulse will go around the cavity, with a decay rate given the losses in the couplers, mirror, splices and attenuation of the fiber. These losses in the cavity were found to be too high, so an erbium-doped fiber amplifier was added to provide enough gain to get a reasonable decay time. The noise was reduced by averaging.

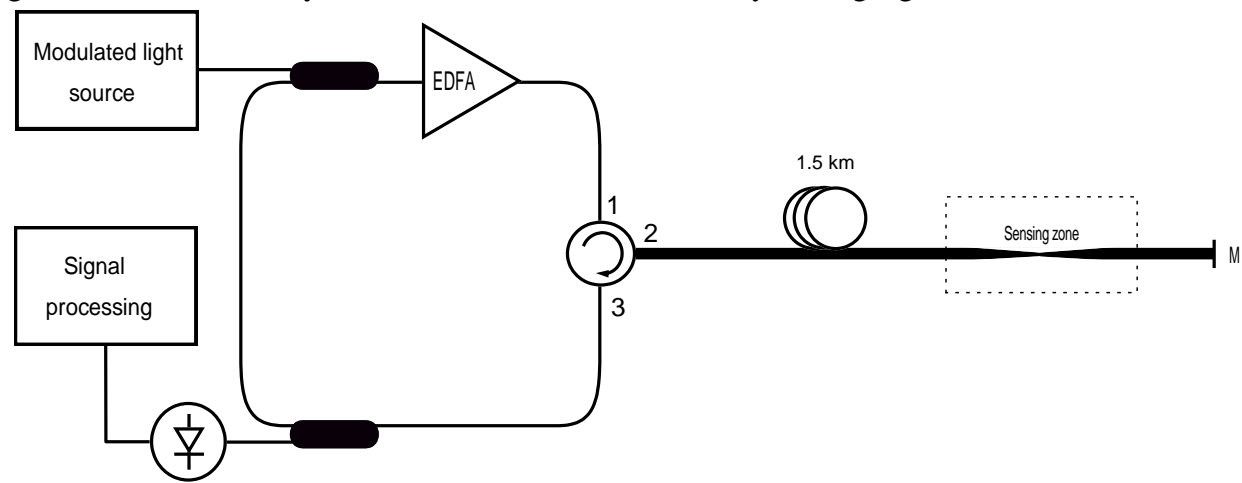

Fig. 1: The experimental setup. A source of optical pulses was coupled to a ring cavity with an erbium-doped fiber amplifier and a circulator. The circulator sends the pulse through over a kilometer of optical fiber, followed by a mirror. The region where a sensing head is added is marked in the figure. 
This configuration was considered for remote sensing (Fig. 1). For this purpose, one could add an intensity-based sensor head after the $1.5 \mathrm{~km}$ of optical fiber and before the mirror. The losses in the sensing head will change the decay time of the pulse in the cavity (the ring-down time). This sensor is easy to implement and allows an application of the cavity ring-down techniques to remote sensing. The placing of a mirror also implies that the pulse will cross the sensor head twice, apparently doubling the sensitivity. On the other hand it also means the pulse will cross the splices twice. Still an improvement in sensitivity might be expected relative to placing it in the ring.

For a proof of concept, a taper was used as a sensor head for displacement (Fig. 2). It was placed before the mirror and, to show the difference in response, also in the ring (at port 3 of the circulator). The taper was glued on a translation stage with a positive displacement here meaning pushing the two ends of the taper closer and increasing the bending. For higher displacements one then obtains higher losses and smaller ring-down times. At $0 \mu \mathrm{m}$ displacement, the taper is extended and there is no loss (besides the splices).

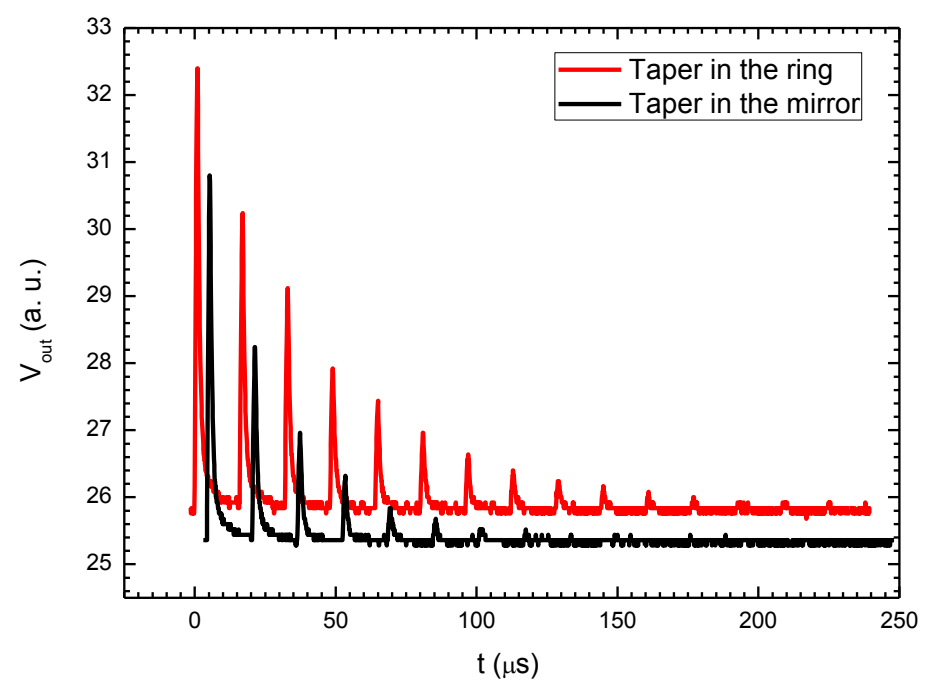

Figure 2: Two decaying pulses in the cavity for the case in which the taper is: after port 3 (red); and after port 2 and $1.5 \mathrm{~km}$ of optical fiber (black). In both cases, the value of the displacement is fixed at $200 \mu \mathrm{m}$.

Figure 2 shows the decay of the pulses in these two configurations, for a fixed value of displacement. The lower value for the baseline when the taper is placed before the mirror comes from the additional loss in the cavity that comes from a splice in the fiber with a mirror being crossed twice. Such loss is independent of the displacement, of course. For the same reason one would expect that for zero displacement the losses are slightly higher, and therefore the ring-down time slightly smaller in the case of the taper placed before the mirror. Such is indeed verified (Fig.3).
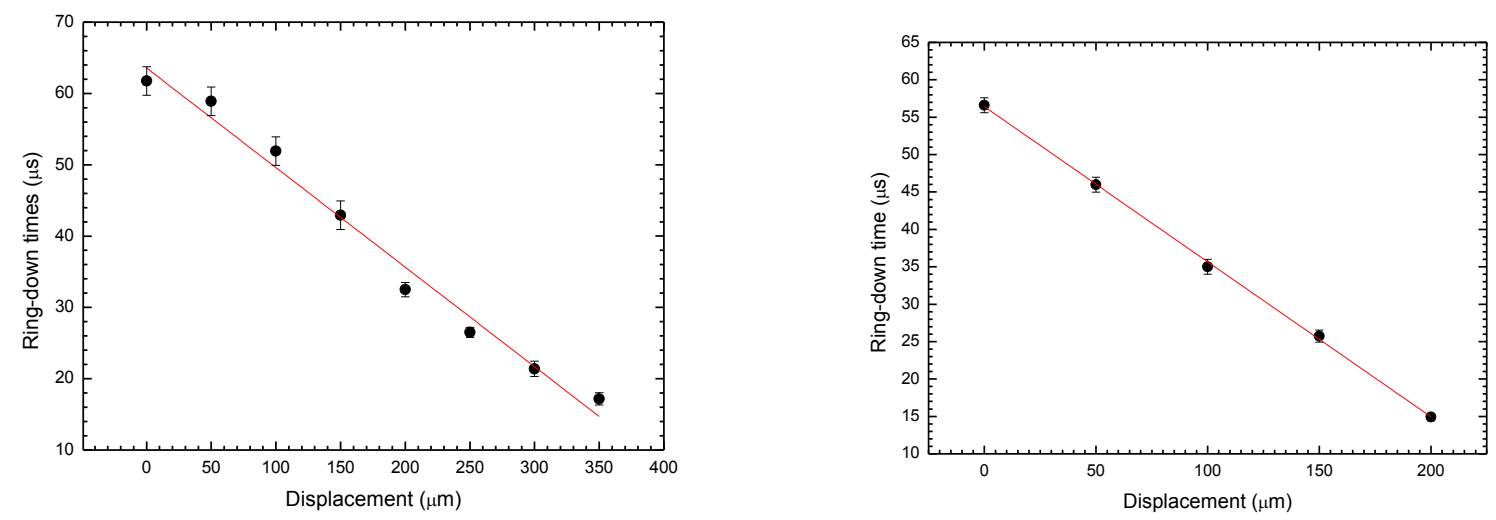

Figure 3: The sensors linear response: in the remote sensing configuration (right), with the taper before the mirror; and with the taper placed in the loop (left), after port 3 of the optical circulator. 
Figure 3 shows very clearly the sensitivity improvement. Zero displacement ring-down times of 56.6 and $61.7 \mu$ s and sensitivities of $0.21 \mathrm{s.m} \mathrm{m}^{-1}$ and $0.14 \mathrm{~s} . \mathrm{m}^{-1}$ were attained for the taper before the mirror and for the taper in the loop, respectively. Since when the ring-down time becomes too small one can no longer perform a reliable exponential fit, higher sensitivity has the drawback of a shorter range of displacements values one can measure. In any case, the results show that cavity ring-down measurements can be performed in remote sensing using this configuration. One should note that the longer the length of fiber at port 2 , the higher the time interval between each peak of figure 2 . To still be able to monitor the decay of the pulse one must then have a greater ring-down time, which could be provided by adjusting the gain of the amplifier.

\section{Conclusion}

A new configuration which allows the application of the CRD technique for remote sensing was proposed. In this topology, the loop, amplifier, couplers and all apparatus required to monitor the signal were kept close to the source of the pulses, with only a length of fiber extending out. A proof of concept was given by using a taper to sense displacement. The difference in response with the taper placed in the remote sensing configuration and in the loop was analyzed.

\section{Acknowledgments}

This work was supported by project "NORTE-07-0124-FEDER-000058" which is financed by the North Portugal, Regional Operational Programme (ON.2 - O Novo Norte), under the National Strategic Reference Framework (NSRF), through the European Regional Development Fund (ERDF).

\section{References}

[1] G. Berden, R. Peeters, and G. Meijer, "Cavity ring-down spectroscopy: Experimental schemes and applications," Int. Rev. Phys. Chem., vol. 19, pp. 565-607, 2000

[2] G. Stewart, K. Atherton, and B. Culshaw, "Cavity-enhanced spectroscopy in fiber cavities," Opt. Lett., vol. 29, no. 5, pp. 442-444, 200

[3] W. P. Tarsa, D. M. Brzozowski, P. Rabinowitz, and K. K. Lehmann, “Cavity ringdown strain gauge,” Opt. Lett., vol. 29, no. 12, pp. 1339-1341, 2004

[4] N. Ni, C. C. Chan, X. Y. Dong, J. Sun, and P. Shum, "Cavity ring-down long period fiber grating strain sensor," Meas. Sci. Technol., Special Issue, vol. 18, pp. 3135-3138, 2007

[5] C. Wang and S. T. Scherrer, "Fiber ringdown pressure sensors," Opt. Lett., vol. 29, no. 4, pp. 352-354, 2004

[6] C. Wang, "Fiber ringdown temperature sensor," Opt. Eng. Lett., vol. 44, no. 3, 030503, 2005

[7] D. J. Passos, S. O. Silva, M. J. Marques, O. Frazão, "Cavity ring-down with OTDR for remote sensing", 23rd Conference on Optical Fiber Sensors, 2014 